\title{
Convective/absolute instability in miscible core-annular flow. Part 1: Experiments
}

\author{
M. d'OLCE, J. MARTIN, N. RAKOTOMALALA, D. SALIN \\ AND L. TALONं \\ Universite Pierre et Marie Curie-Paris 6, Universite Paris-Sud, CNRS, F-91405, \\ Lab FAST, Bat 502, Campus Universitaire, Orsay F-91405, France
}

(Received 7 December 2007 and in revised form 15 September 2008)

We address the issue of the convective or absolute nature of the instability of coreannular pipe flows, in experiments using two miscible fluids of equal density but different viscosities, the core fluid being much less viscous than the wall one. We use a concentric co-current injection of the two fluids. An axisymmetric parallel base state is obtained downstream the injector. The core radius $R_{I}$ and the Reynolds number $R e$ of the so-obtained base state are varied independently due to the control of the flow rate of each fluid. However, a downstream destabilization of this base state was observed within the explored range of the two control parameters $R_{I}$ and $R e$. Moreover, the fixed location of this destabilization, observed for some particular parameters, suggests an absolute nature of the instability. We present a tentative delineation of the nature (convective or absolute) of the instability and discuss the accessible measurements to experimentally address this issue.

\section{Introduction}

First discovered in the context of plasma physics (Briggs 1964), the concept of absolute and convective instability has been significantly used in hydrodynamic open flows (Huerre \& Monkewitz 1990) such as a falling film down an inclined wall (Brevdo, Laure \& Bridges 1999) or a fibre (Duprat et al. 2007), wakes (Chomaz 2005). When a given unstable flow is locally perturbed by a small disturbance, two different types of evolution can be encountered (Huerre \& Monkewitz 1990). For a convectively unstable flow (CU), the disturbances are amplified and advected away from their initial location. Such a flow behaves like a noise amplifier. For an absolutely unstable flow (AU), although advected, the perturbation is so strongly amplified that it invades the whole space (downstream and upstream). In such a case, the so-called global mode will prevail at long times and the system will behave like a self-sustained resonator, which oscillates at an intrinsic frequency (Huerre \& Rossi 1998; Chomaz 2005). In a theoretical frame, the transition corresponds to the pinching of two spatial branches of the dispersion equation. Therefore, the transition can be either computed or obtained by the study of the impulse response as carried out in the companion paper (Selvam et al. 2008). However, except in devoted experiments where artificial perturbations have been introduced by means of a magnetically driven vibrating ribbon (Huerre \& Monkewitz 1990) or in the case of a subcritical transition (Gondret et al. 1999), the transition is experimentally difficult to determine (Duprat et al. 2007). Indeed, it is non-trivial to perturb the system at a particular location away from the boundary conditions. Moreover, in the absolute case, the base state is experimentally

$\dagger$ Email address for correspondence: talon@fast.u-psud.fr 


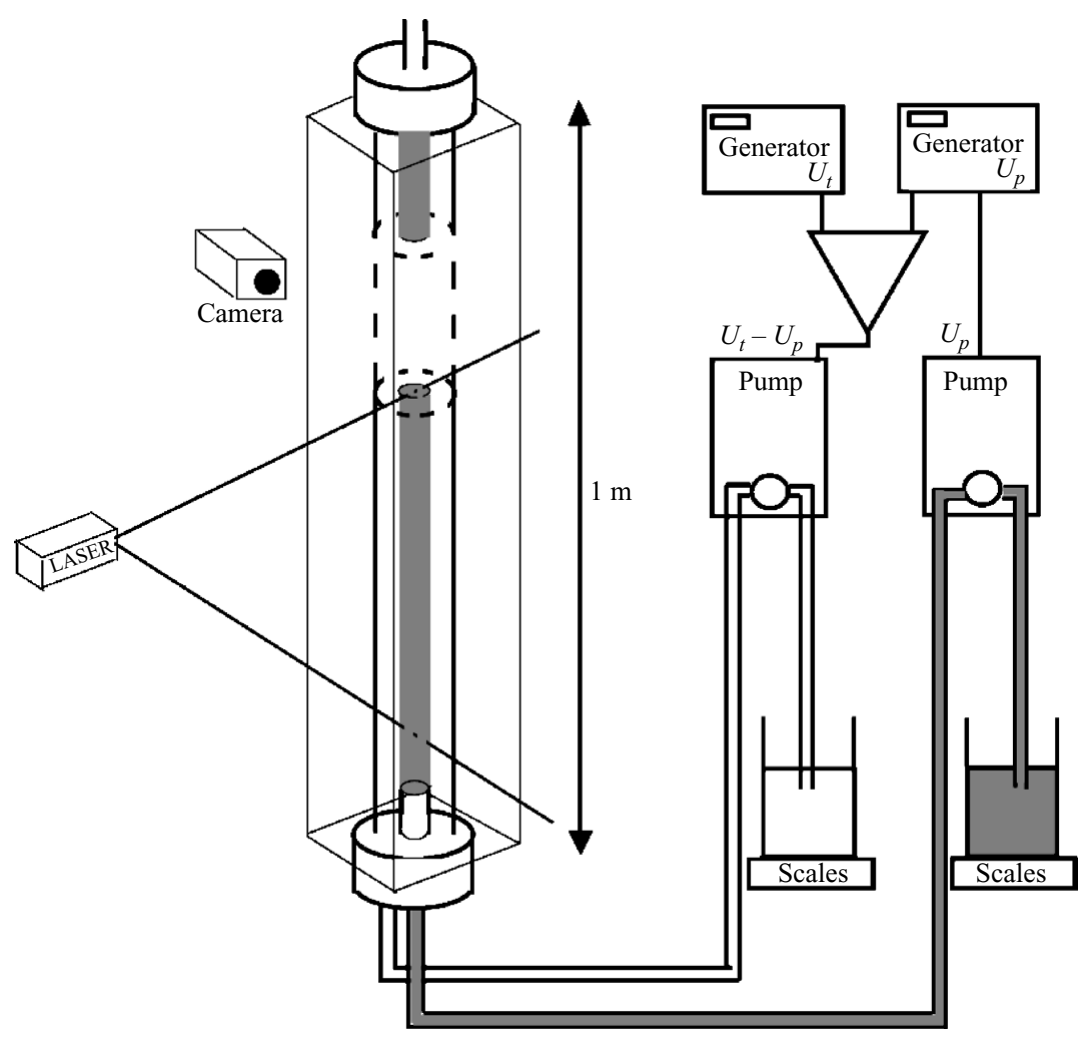

FIGURE 1. Sketch of the experimental setup used to achieve a core-annular flow. One metre long cylindrical tube has a radius $R=1 \mathrm{~cm}$. The core fluid is injected in the smaller tube of inner radius $0.75 \mathrm{~cm}$ and outer radius $0.85 \mathrm{~cm}$. The wall fluid is injected in the remaining annular, between the smaller tube and the wall. The flow rate of each fluid is monitored by a pump.

unachievable as the presence of some noise, which may trigger the instability, cannot be avoided.

Here, we address the issue of the experimental delineation of the CU/AU transition for the case of the core-annular flow (CAF) of two miscible non-buoyant fluids, as sketched in figure 1. Note that miscible CAFs are used to feed some static mixers, which lose efficiency when axial segregation of the fluids occurs upstream the mixer, which may happen in the case of absolute instabilities (Cao et al. 2003).

The instabilities of CAFs have been widely investigated in the case of immiscible fluids (Hickox 1971; Joseph, Renardy \& Renardy 1984; Hu \& Joseph 1989; Bai, Chen \& Joseph 1992; Joseph \& Renardy 1992a,b; Joseph et al. 1997; Kouris \& Tsamopoulos 2001, 2002; Guillot et al. 2007). They involve competition between viscous shear, inertia, capillarity and buoyancy and can be enlightened by the analysis of the two-dimensional shear flows, as performed by Hinch (1984), Albert \& Charru (2000) and Charru \& Hinch (2000). Taking into account buoyancy and surface tension, Hickox (1971) showed that a CAF with the less viscous fluid in the core was unstable. Later, Joseph et al. (1984) showed that the CAF configuration with the less viscous fluid at the wall, used to lubricate pipeline flows, was unstable if the annular film was not thin enough. These early works have been completed by the study of the nonlinear effects (Papageorgiou, Maldarelli \& Rumschitzki 1990; Kouris 
\& Tsamopoulos 2001, 2002). More recently, Guillot et al. (2007) have studied the lubrication regime of the CAF for microfluidic applications. They have shown that the $\mathrm{CU} / \mathrm{AU}$ transition could be invoked to explain the onset of the drop-producing regime (allowing various applications as emulsification and encapsulation).

For the miscible case, most effort has been paid to characterize the influence of the viscosity ratio and of the buoyancy effects on the displacing front (Chen \& Meiburg 1996; Petitjeans \& Maxworthy 1996; Scoffoni, Lajeunesse \& Homsy 2001; Kuang, Maxworthy \& Petitjeans 2003; Balasubramaniam et al. 2005). Miscible fluids are usually used under conditions such that the two fluids have not time enough to mix, i.e. at high Péclet number. The fluids therefore exhibit a sharp pseudo-interface between them, but without surface tension. The issue of the comparison of the miscible fluid displacement in the limit of large Péclet numbers with the immiscible fluid one in the limit of large capillary numbers has been addressed (Petitjeans \& Maxworthy 1996): In these two limits, the flows of miscible fluids without diffusion are similar to the flows of immiscible fluids without surface tension. In addition, we note that the lack of surface tension in miscible flows leads to a configuration similar for two-dimensional flows and for axisymmetric ones. Recently, Selvam et al. (2007) have performed the temporal linear stability analysis of the non-buoyant miscible coreannular flow instabilities, using spatially periodic perturbations. They have shown that such a flow was subject to axisymmetric instabilities at high Schmidt number. However, this temporal analysis does not address the issue of the convective/absolute nature of the instability which characterizes open flows, where perturbations develop spatially.

In the present paper, we study experimentally the effect of the Reynolds number and of the core fluid radius on the convective/absolute nature of the instability for a fixed viscosity ratio. In $\S 2$, we present the experimental setup. In $\S 3$, we introduce all the different measurements used to investigate the instability and the CU/AU transition presented in $\S 4$. A discussion of the results follows in $\S 5$. In a following companion paper (Selvam et al. 2008), our results will be quantitatively discussed and compared to the linear stability analysis and nonlinear numerical simulations.

\section{Experimental setup}

\subsection{Core-annular flow setup}

The experimental setup is sketched in figure 1. Experiments are performed in a vertical transparent Perspex cylindrical tube of length $1 \mathrm{~m}$ and internal radius $R=1 \mathrm{~cm}$. An upwards injection of the two fluids is implemented, using a concentric inner nozzle tube of inner and outer radii 0.75 and $0.85 \mathrm{~cm}$. The two fluids, namely the core fluid and the wall fluid, are simultaneously injected with two pumps, at the constant flow rates, $Q_{\text {core }}$ and $Q_{\text {wall }}$, respectively. Note that for the experiments of harmonic excitation $(\$ 4.2)$, an electronic device enables to impose an oscillating $Q_{\text {core }}$, while maintaining the total flow rate $Q=Q_{\text {core }}+Q_{\text {wall }}$ constant. The two fluids are salted water for the core and a water-natrosol mixture for the wall, of viscosities equal to $\eta_{\text {core }}=10^{-3} \mathrm{~Pa} \cdot \mathrm{s}$ and $\eta_{\text {wall }}=(25 \pm 2) \times 10^{-3} \mathrm{~Pa} \cdot \mathrm{s}$, respectively. Salt (calcium chloride) was added to match the densities of the two fluids to $\rho=1000 \mathrm{~kg} \mathrm{~m}^{-3}$, with an accuracy of $\pm 10^{-1} \mathrm{~kg} \mathrm{~m}^{-3}$. These mixtures are perfectly Newtonian in the range of shear rates used in our experiments (Torrest 1982; Le Bars \& Davaille 2002), and have a viscosity ratio $M=\log \left(\eta_{\text {wall }} / \eta_{\text {core }}\right)=3.2 \pm 0.1$. To ascertain the validity of our results, some additional experiments were also performed with water-glycerol mixtures with the same viscosity ratio. 
(a)

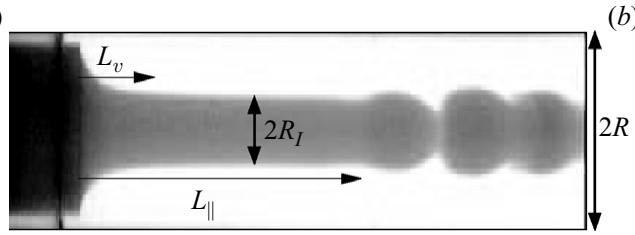

(b)

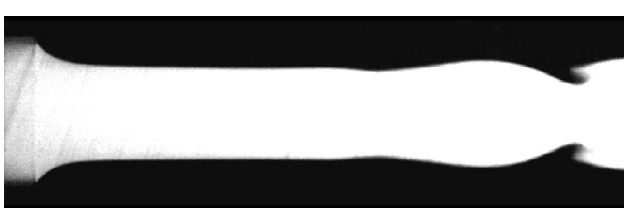

FIGURE 2. (a) Instability pattern for a core fluid relative radius $\tilde{R}_{I}=0.37$, with the core fluid dyed blue. ( $b$ ) Instability pattern for a core fluid relative radius $\tilde{R}_{I}=0.48$, with the core fluid dyed with fluorescin, obtained using a laser sheet. The well-defined pseudo-interface between the two fluids $\tilde{r}_{I}(x, t)$ is determined from these images. We can observe streamwise from the inlet: (1) the viscous entry length $L_{v}$, of the order of the tube radius; (2) a quasi-parallel length $L_{\|}$where the pseudo-interface between fluids looks parallel; (3) an axisymmetric instability pattern, the shape of which is mushroom like $\left(\mathrm{e}^{M}=25, R e=20\right)$.

As can be seen in figure 2, a parallel core-annular flow is achieved beyond an inlet length $L_{v}(\sim 1 \mathrm{~cm}$ in our experiments). This length results from the competition between advection and diffusion of mass momentum. The core radius in the parallel flow region is set by the ratio $Q_{\text {core }} / Q$. Indeed, under the parallel flow conditions and assuming that the two fluids do not mix, the base Poiseuille parabolic-like velocity profile expressed in terms of the core fluid relative radius $\tilde{R}_{I}=R_{\text {core }} / R$ and the relative radial coordinate $\tilde{r}=r / R$ reads

$$
U_{\text {wall }}=\frac{2\left(1-\tilde{r}^{2}\right)}{1+\left(\mathrm{e}^{M}-1\right) \tilde{R}_{I}{ }^{4}} \bar{U}
$$

for the wall fluid $\left(\tilde{R}_{I}<\tilde{r}<1\right)$ and

$$
U_{\text {core }}=\frac{2\left(1+\left(\mathrm{e}^{M}-1\right) \tilde{R}_{I}^{2}-\mathrm{e}^{M} \tilde{r}^{2}\right)}{1+\left(\mathrm{e}^{M}-1\right) \tilde{R}_{I}^{4}} \bar{U}
$$

for the core fluid $\left(0<\tilde{r}<\tilde{R}_{I}\right)$, where $\bar{U}=Q /\left(\pi R^{2}\right)$ is the average flow velocity. Thus, the core fluid relative flow rate reads

$$
\tilde{Q}_{\text {core }}=\frac{Q_{\text {core }}}{Q}=\frac{2 \tilde{R}_{I}^{2}+\left(\mathrm{e}^{M}-2\right) \tilde{R}_{I}^{4}}{1+\left(\mathrm{e}^{M}-1\right) \tilde{R}_{I}^{4}} .
$$

As the above function is monotonic for $\tilde{R}_{I} \in[0,1]$, the core radius is uniquely determined from the flow ratio $\tilde{Q}_{\text {core }}$. Here, we will use $\tilde{R}_{I}$ as a control variable, together with the Reynolds number, defined by $R e=\rho \bar{U} R / \eta_{\text {core }}$. We explored the ranges $\tilde{R}_{I} \in[0,0.6]$ and $R e \in[2,60]$, for the fixed viscosity ratio $\eta_{\text {wall }} / \eta_{\text {core }}=\mathrm{e}^{M}=25$. We note that our experiments were performed with a fixed Schmidt number $S c=\eta_{\text {core }} / \rho D_{m}$. The molecular diffusion coefficient $D_{m}$ estimated as in Torrest (1982) and Le Bars \& Davaille (2002) is $D_{m} \simeq 10^{-10} \mathrm{~m}^{2} \mathrm{~s}^{-1}$, and thus $S c \sim 10^{4}$. Since the Péclet number reads $P e=\bar{U} R / D_{m}=S c R e$, the control parameter $R e$ also quantifies the relative magnitude of convective and diffusive effects. As a consequence, keeping a well-defined interface requires a high enough $P e$, and thus $R e$ ( $R e>3$ in our case). On the other hand, for either high $R e$ values $(R e>60)$ or high $\tilde{R}_{I}$ values $\left(\tilde{R}_{I}>0.6\right)$, the instability patterns exhibit some asymmetry. One may note that in our range of $\tilde{R}_{I}$, the difference between the shears on both sides of the interface, $\Delta \dot{\gamma}=\left(4 r\left(\mathrm{e}^{M}-1\right) \bar{U}\right) /\left(1+\left(\mathrm{e}^{M}-1\right) r^{4} R\right)$, is almost linear in $r$ 
(a)

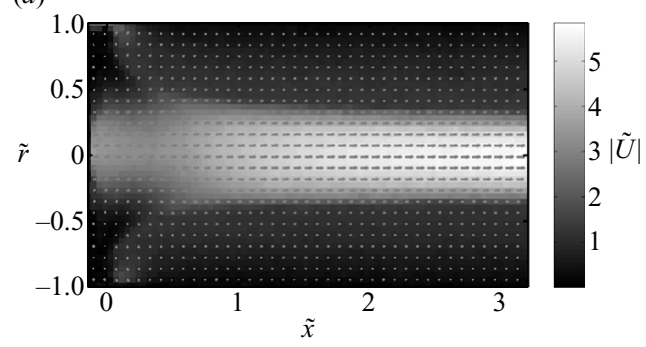

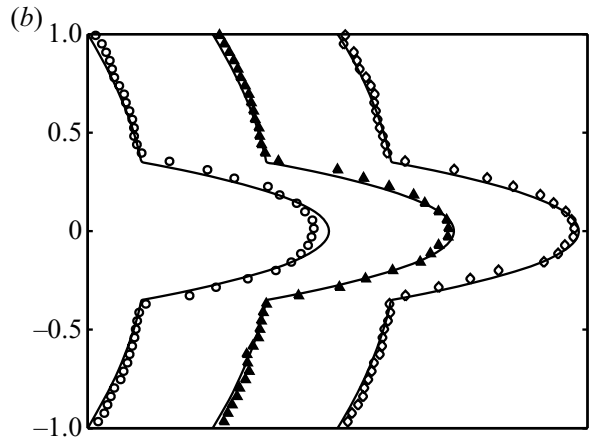

FIGURE 3. PIV measurements of the velocity field in the vicinity of the inlet across the whole tube diameter. (a) Velocity field. The direction of the velocity is indicated by an arrow and its intensity is coded in grey scale. Note that the pseudo-interface contour between the two fluids is barely visualized. $(b)$ Three transverse velocity profiles measured at distances $2.2,2.7,3.2 \mathrm{~cm}$ from the inlet. For clarity, the profiles have been shifted. The lines through the data correspond to the theoretical Poiseuille velocity profile obtained for parallel flows of two non-mixing fluids $\left(\mathrm{e}^{M}=25, \operatorname{Re}=14, \tilde{R}_{I}=0.36\right)$.

(for $r^{4}<1 /\left(3\left(\mathrm{e}^{M}-1\right)\right)$, i.e. $r<0.35$ for $\left.\mathrm{e}^{M}=25\right)$, so that $r$ quantifies also the shear difference, $\Delta \dot{\gamma} \simeq 4 r\left(\mathrm{e}^{M}-1\right) \bar{U} / R$, in most of our experiments.

Here, we will use characteristic length and time $R$ and $R / \bar{U}$, respectively, to define non-dimensionalized physical quantities (marked with a tilde).

\subsection{Optical setup}

\subsubsection{Concentration measurement}

Measurements were achieved using a video camera. To minimize any optical distortion induced by a variation of optical indices between the tube and the atmosphere, we enclosed the apparatus in a square box filled with water. We observed a small magnifying effect with no distortion. Preliminary experiments were performed using a dyed blue core fluid (figure $2 a$ ). However, to analyse more precisely the pseudo-interface between the two fluids, we used fluorescin as a dye and illuminated the axis of the tube with a thin $1 \mathrm{~mm}$ wide laser sheet as sketched in figure 1 and shown in figure $2(b)$. This technique provides a measurement of the interface radius $\tilde{r}_{I}(\tilde{x}, \tilde{t})$ with an accuracy better than $5 \%$. In these figures, we can observe streamwise from the inlet: (1) the viscous entry length $L_{v}$, of the order of the tube radius; (2) a quasi-parallel length $L_{\|}$where the pseudo-interface between the fluids is parallel; (3) an axisymmetric instability pattern, the shape of which is mushroom like. We have already reported on these nonlinear axisymmetric patterns (d'Olce et al. 2008).

\subsubsection{PIV measurements of the velocity profile}

In order to measure the flow field in the inlet region, we used the Particle Image Velocimetry (PIV) software (LaVision GmbH, Goettingen, Germany). The camera has a resolution of $1280 \times 608$ pixels in the plane of the laser sheet (figure 1). The PIV algorithm requires a window of $32 \times 32$ pixels. Measurements over the whole cell diameter $(2 \mathrm{~cm})$ provide typically 20 data points across the tube section. The spatial resolution is therefore of the order of $1 \mathrm{~mm}$. Figure $3(a)$ gives a typical velocity field. Figure $3(b)$ displays three velocity profiles $\tilde{U}(\tilde{r})$ at three locations from the inlet $(2.2$, 2.7 and $3.2 \mathrm{~cm}$ ). The three solid lines correspond to the theoretical velocity profile (2.1) and (2.2). The agreement of the latter with the measurements supports the contention 
that a parallel flow is achieved and that the diffusive mixing is actually negligible. The latter statement agrees with the estimation of the pseudo-interface thickness (smaller than $0.03 \mathrm{~cm}$ ) obtained from figure $2(b)$.

\section{Experimental observations and quantities of interest}

In this section, we introduce the different quantities that will be used to investigate the CU/AU transition.

\subsection{Interface spatio-temporal diagram}

In the axisymmetric patterns (see figure $2 b$ ), the inflexion point of the light intensity provides the space- and time-dependent radial position of the pseudo-interface $\tilde{r}_{I}(\tilde{x}, \tilde{t})$. In figure $4(a)$, we plot the time dependence of $\tilde{r}_{I}(\tilde{x}, \tilde{t})$ for four different locations $\tilde{x}$. As expected for a spatially unstable regime, we observe, downstream from the inlet, an increase of the amplitude of the fluctuations. It is convenient to plot these variations $\tilde{r}_{I}(x, t)$ on a spatio-temporal diagram: The vertical axis is the time $\tilde{t}$, the horizontal axis the distance $\tilde{x}$ from the inlet and the position $\tilde{r}_{I}(x, t)$ of the interface is encoded into a grey level. A typical spatio-temporal diagram is displayed in figure $4(b)$. The homogeneous grey level close to the inlet corresponds to a region of constant $\tilde{r}_{I}$ (quasi-parallel flow base state). Further downstream, one can observe dark and light stripes, the slope and contrast of which give the velocity and the amplitude of the instability waves.

\subsection{Temporal power spectrum of the interface fluctuations}

As mentioned above, a key difference between convective and absolute states is their spatial response to noise. Therefore, it may be useful to analyse the spatial evolution of the frequency spectrum with $\tilde{x}$. For that purpose, we decompose at each location $\tilde{x}$ the temporal fluctuations of $\tilde{r}_{I}(t)$ into Fourier modes characterized by their amplitude and their phase, i.e. $A(\tilde{x}, \tilde{f}) \mathrm{e}^{\mathrm{i}(\phi(\tilde{x}, \tilde{f})-2 \pi \tilde{f} \tilde{t})}$. From this formulation, one can compute the local wavenumber $\tilde{k}_{r}(\tilde{x}, \tilde{f})=\partial \phi / \partial \tilde{x}$ and the local wave speed $\tilde{c}(\tilde{x}, \tilde{f})=2 \pi \tilde{f} / \tilde{k}_{r}(\tilde{x}, \tilde{f})$ for each mode.

A typical frequency power spectrum $A^{2}(\tilde{x}, \tilde{f})$ measured at a given $\tilde{x}$ position, is plotted in figure $4(c)$. Such a frequency distribution is characterized by the frequency at which the distribution is maximum $\tilde{f}_{\max }(\tilde{x})$ and by the spectrum width measured by the root mean square deviation from the mean, r.m.s. $(\tilde{x})$ :

$$
\begin{array}{r}
r . m . s .(\tilde{x})=\sqrt{\int p(\tilde{x}, \tilde{f}) \tilde{f}^{2} \mathrm{~d} \tilde{f}-\left(\int p(\tilde{x}, \tilde{f}) \tilde{f} \mathrm{~d} \tilde{f}\right)^{2}} \\
\text { with } p(\tilde{x}, \tilde{f})=A^{2}(\tilde{x}, \tilde{f}) / \int A^{2}(\tilde{x}, \tilde{f}) \mathrm{d} \tilde{f} .
\end{array}
$$

Figure $4(d)$ gives an example of the spatial evolution of the maximum amplitude of the power spectrum, $A_{m}^{2}(\tilde{x})$. One can observe an increase of the amplitude followed by its saturation. Close enough from the inlet, the spatial growth appears to be exponential, revealing a linear regime. We will measure the characteristics of the instability patterns in this linear range, namely the spatial growth rate $-\tilde{k}_{i}$, the phase velocity $\tilde{c}$ and the real part of the wavenumber $\tilde{k}_{r}$.

A typical spatial evolution of the root mean square deviation is plotted in figure $4(e)$. Near the inlet, no instability is detectable, so the spectrum is a random noise one (infinite r.m.s.), and the measured r.m.s. is fixed by the size of the measurement window. Downstream, the r.m.s. decreases as the instability grows and reaches a 
(a)
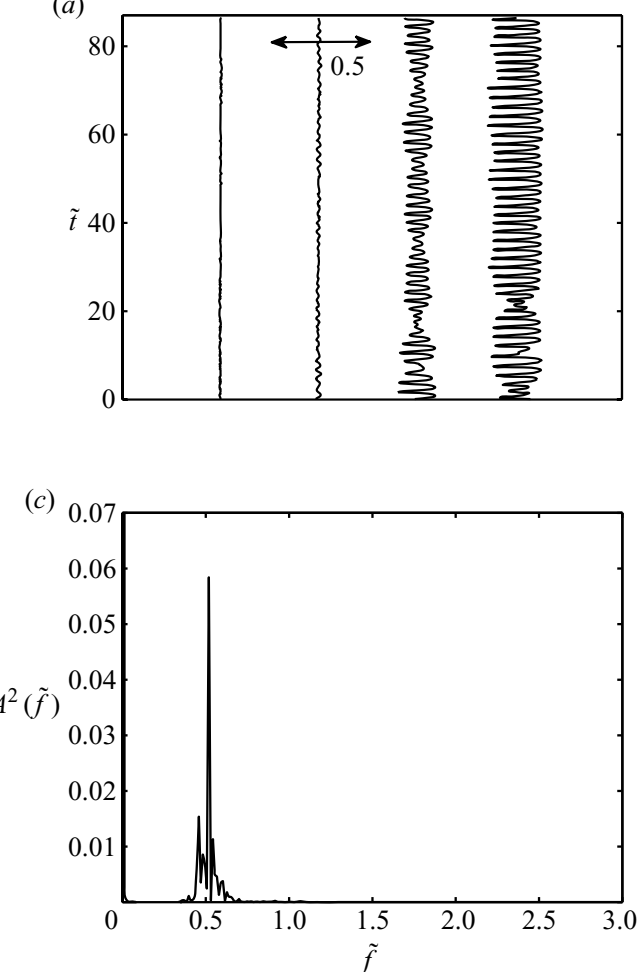

(e)

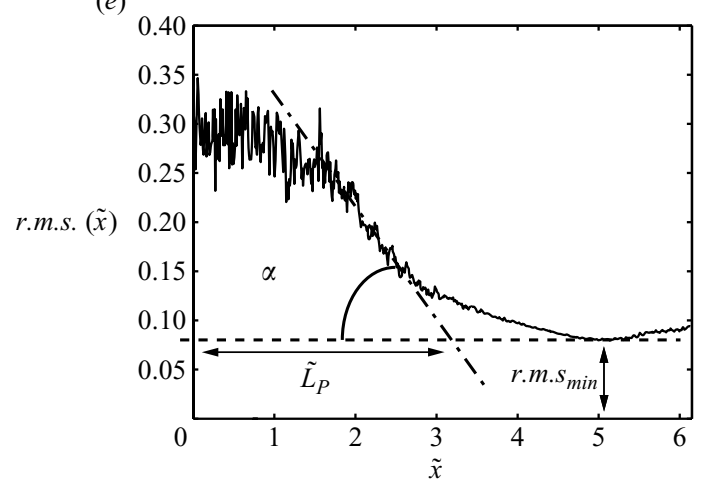

(b)

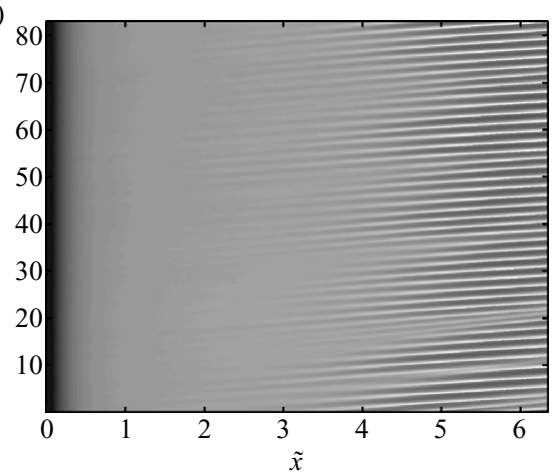

(d)
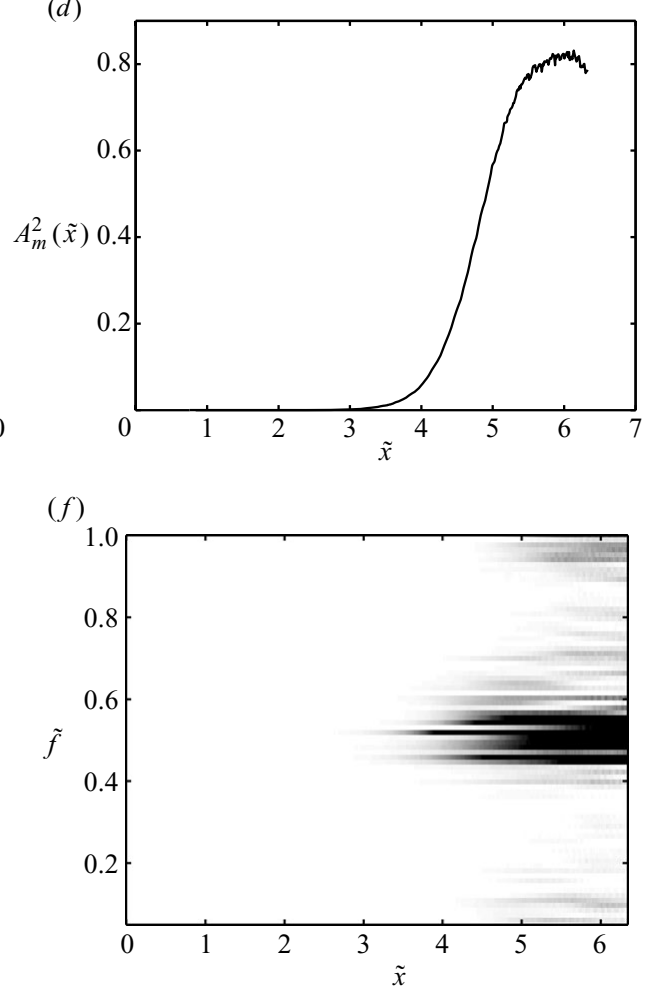

Figure 4. (a) Time $(\tilde{t})$ variations of the interface location $\tilde{r}_{I}(\tilde{x}, \tilde{t})$ at four different distances from the inlet, $\tilde{x}=1.5,3,4.5,6$. For clarity, the mean position $\tilde{R}_{I}$ has been shifted along the horizontal axis. (b) Spatio-temporal diagram. The vertical axis is the time $\tilde{t}$, the horizontal axis is the distance $\tilde{x}$ from the inlet and the grey level corresponds to the position $\tilde{r}_{I}(\tilde{x}, \tilde{t})$ of the interface. (c) Power spectrum. Square of the amplitude of the modes $A^{2}(\tilde{f})$ versus their frequency $\tilde{f}$, of the Fourier transform of the temporal variations of the interface at $\tilde{x}=4$. The frequency at which the distribution is maximum is denoted $\tilde{f}_{\max }(\tilde{x})$. $(d)$ Square of the amplitude of the frequency $\tilde{f}_{\max }(\tilde{x}), A_{m}^{2}(\tilde{x})$, versus the distance $\tilde{x}$ from the inlet. The spatial growth rate is measured by an exponential fit of the linear regime. (e) R.m.s. of the power spectrum, r.m.s.( $\tilde{x})$, versus $\tilde{x}$ and definition of the minimum of the r.m.s., r.m.s.min, of the filtering length $\tilde{L}_{p}$ and of the spatial rate of filtering $\alpha$. $(f)$ Frequency $\tilde{f}$ versus $\tilde{x}$ diagram where the amplitude of the power spectrum is coded in grey scale $\left(\mathrm{e}^{M}=25, R e=20, \tilde{R}_{I}=0.48\right)$. 
minimum value, denoted by r.m.s.min, at large $\tilde{x}$. Since a CU flow behaves like a noise amplifier and because our inherent noise is broadband, we can expect that the CU state will have a r.m.s.min larger than the one of an AU state which resonates at a single frequency.

To account for the spatial variations of these spectra, we built a frequency $\tilde{f}$ versus space $\tilde{x}$ diagram where the amplitude of the power spectrum is encoded in grey scale. Such a typical diagram is given in figure $4(f)$. Like in the spatio-temporal diagram, one can observe that a minimum distance from the inlet is required for the most unstable mode to be amplified. One can also note, further downstream, the appearance of the corresponding harmonic modes.

\subsection{Quasi-parallel length $\tilde{L}_{\|}$, filtering length $\tilde{L}_{P}$ and filtering rate $\alpha$}

An important feature of the observed instability pattern is the presence of a parallel flow region at distances from the inlet between the viscous entry length $L_{v}$ and the position where the instability appears. The extension of this region might be a relevant feature to discriminate the nature of the instability. Indeed, in a very precise theoretical context (no background noise in a semi-infinite domain), this region is expected to extend over an infinite or finite length for a CU or an AU instability, respectively (see Couairon \& Chomaz 1997a, b). In our experimental context, in which background noise cannot be avoided, this parallel region remains finite in any case. In order to evaluate it, we compute, for each location $\tilde{x}$, the time average of the magnitude of the interface oscillation. We define the quasi-parallel length $\tilde{L}_{\|}$as being the location at which this average magnitude is equal to a given threshold.

An alternative characterization of the parallel region can be obtained from the plot of the r.m.s. of the frequency power spectrum versus $\tilde{x}$, r.m.s.( $\tilde{x}$ ) (figure $4 e$ ). We denote by $\tilde{L}_{P}$ the intersection location of the tangent at the inflexion point with the minimum of the r.m.s. Physically, as r.m.s.min evaluates the inverse of the quality factor of the noise filter, $\tilde{L}_{P}$ quantifies the distance needed for the filter to be efficient. The latter may indeed be different from the length needed for the instability to appear, $\tilde{L}_{\|}$. One may note that $\tilde{L}_{\|}$and $\tilde{L}_{P}$ are defined as distances from the inlet nozzle $(\tilde{x}=0)$, which does not match at all the theoretical semi-infinite domain origin (i.e. a location with a non-noisy unstable basic state).

Another characterization of the spatial decrease of r.m.s.( $(\tilde{x})$, which is not linked to the inlet location, may be obtained through the value of the slope at the inflexion point as sketched in figure 4(e). The maximum slope of r.m.s. $(\tilde{x}), \alpha$, will be called the filtering spatial rate. We note that $-1 / \alpha$ is a typical length, which should compare with $\tilde{L}_{P}$ but which is measured locally.

We have already pointed out the differences between the theoretical frame of work and the experimental constraints which may raise difficulties in delineating the change of nature of the instability. However, since an AU system behaves like a frequency resonator and since global modes saturate at a finite distance from the inlet, we expect, for a background noise small enough, that r.m.s.min $, \tilde{L}_{P}, \tilde{L}_{\|}$and $\alpha$ drastically change from a $\mathrm{CU}$ to an $\mathrm{AU}$ state.

\section{Delineation of the transition between the convectively and absolutely unstable states}

In this section, we shall first measure the characteristics of our experimental instability, as defined in the previous section. Then, the response of the system to a harmonic perturbation will be analysed. 


\subsection{Natural instability}

The two control parameters of the experiment are the Reynolds number $R e$ and the relative core radius $\tilde{R}_{I}$ of the quasi-parallel base state. As mentioned above, the interface is well defined and the patterns remain axisymmetric in limited ranges of these two parameters, $\tilde{R}_{I} \in[0,0.6]$ and $R e \in[2,60]$. In these ranges, the parallel core-annular flow never extended all along the tube, whatever attention paid to the injection conditions. We have investigated various features of the instability which develops naturally in the system, in the parameter space $\left(\tilde{R}_{I}, R e\right)$. For the sake of clarity, we will first analyse the evolution of our different measurements, with one of the two control parameters fixed $\left(R e=48\right.$ and $\tilde{R}_{I}=0.48$ for the left column and right column, respectively, of the graphs of figures 5 and 6). The variations of the quantities of interest in the plane $R e-\tilde{R}_{I}$ are then discussed and displayed in figure 7.

Figure 5 displays the measured frequency $\tilde{f}_{\text {max }}$, wavenumber $\tilde{k}_{r}\left(\tilde{f}_{\text {max }}\right)$ and phase velocity $\tilde{c}\left(\tilde{f}_{\text {max }}\right)$ of the most amplified mode. The latter decreases continuously with $\tilde{R}_{I}$ (figure $5 e$ ) whereas it remains almost constant as $R e$ is varied (figure $5 f$ ). Moreover, the measurements of $\tilde{c}\left(\tilde{f}_{\max }\right)$ compare very well with the phase velocity $\tilde{c}_{S W}$ (dashed lines in figures $5 e$ and $5 f$ ) obtained in the short wave limit of the temporal linear stability analysis (Joseph \& Renardy 1992b). The latter equals the fluid velocity at the interface in the parallel base state and can be obtained with (2.1) or $(2.2): \tilde{c}_{S W}=\tilde{U}_{\text {wall }}\left(\tilde{R}_{I}\right)=\tilde{U}_{\text {core }}\left(\tilde{R}_{I}\right)=\left(2\left(1-\tilde{R}_{I}^{2}\right)\right) /\left(1+\left(\mathrm{e}^{M}-1\right) \tilde{R}_{I}^{4}\right)$. We note that in our experiments, this expression of $\tilde{c}_{S W}$ is found to hold for the phase velocity of the most amplified mode, despite the not very large values of the normalized wavenumber $\tilde{k}_{r}\left(\tilde{f}_{\max }\right)$ (between 3 and 10, typically). Thereby, the frequency and the wavenumber of the most amplified mode are linked by the relation $2 \pi \tilde{f}_{\max } / \tilde{k}_{r}\left(\tilde{f}_{\text {max }}\right)=\tilde{c}_{S W}\left(\tilde{R}_{I}\right)$. As a matter of fact, the frequency $\tilde{f}_{\text {max }}$ and the wavenumber $\tilde{k}_{r}\left(\tilde{f}_{\text {max }}\right)$ do increase proportionally (by a factor 2) as $R e$ is varied from 5 to 50 , keeping $\tilde{R}_{I}=0.48$ constant (right column of figure 5 ). It has to be noted that the ratio $2 \pi \tilde{f}_{\max } / \tilde{k}_{r}$, obtained by a linear stability analysis and direct numerical simulations by Selvam et al. (2008), was also found to be independent of $R e$, although slightly higher than $\tilde{c}_{S W}$. Conversely, as the core radius is increased at a fixed $R e=48$ (left column of figure 5), the frequency exhibits a strong decrease whereas the wavenumber remains nearly constant. In other words, the rather strong variations of the phase velocity, $\tilde{c}\left(\tilde{f}_{\max }\right)=\tilde{c}_{S W}\left(\tilde{R}_{I}\right)$, with the core radius are accounted by the frequency variations. To summarize, the wavenumber of the most amplified mode is fixed by the Reynolds number whereas its phase velocity is set by the core radius. And despite a small jump in $\tilde{f}_{\text {max }}$ and $\tilde{k}_{r}\left(\tilde{f}_{\text {max }}\right)$ in the vicinity of $\tilde{R}_{I} \sim 0.45$ and $R e \sim 45$, we do not observe any drastic change in the most amplified mode which could characterize a change in the instability nature.

To address the issue of the nature of the instability, we have defined quantities which are expected to be more discriminating, namely r.m.s.min $, \tilde{L}_{P}, \tilde{L}_{\|}$and $\alpha$. Figure 6 displays the following:

$(a, b)$ : The minimum of the frequency r.m.s., r.m.s.min, which characterizes the oscillator at work. The frequency distribution width decreases significantly with both $\tilde{R}_{I}$ and $R e$ and levels off at a value corresponding to the minimum measurable width of our frequency window $(\sim 0.02)$, for $R_{I} \gtrsim 0.45$ and $R e \gtrsim 40$, respectively.

$(c, d)$ : The quasi-parallel length $\tilde{L}_{\|}(\triangle)$ and the filtering length $\tilde{L}_{P}(\bullet)$. The two lengths exhibit the same trend: A strong decrease from about 7 to a value smaller than 1 as either $\tilde{R}_{I}$ or $R e$ is increased. Note that the minimum measured values are smaller than 1, which is of the order of the viscous length. In other words, both lengths are so small that the instability develops before the parallel state is completely achieved. 


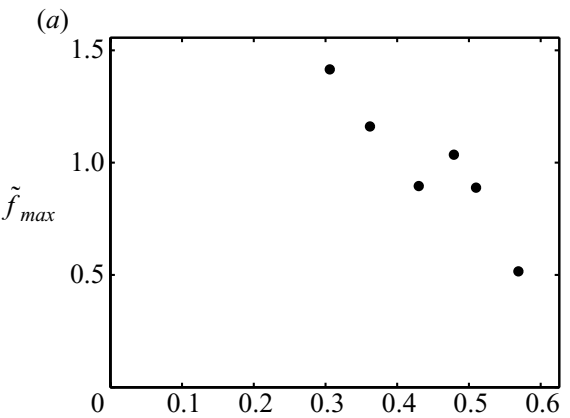

(b)
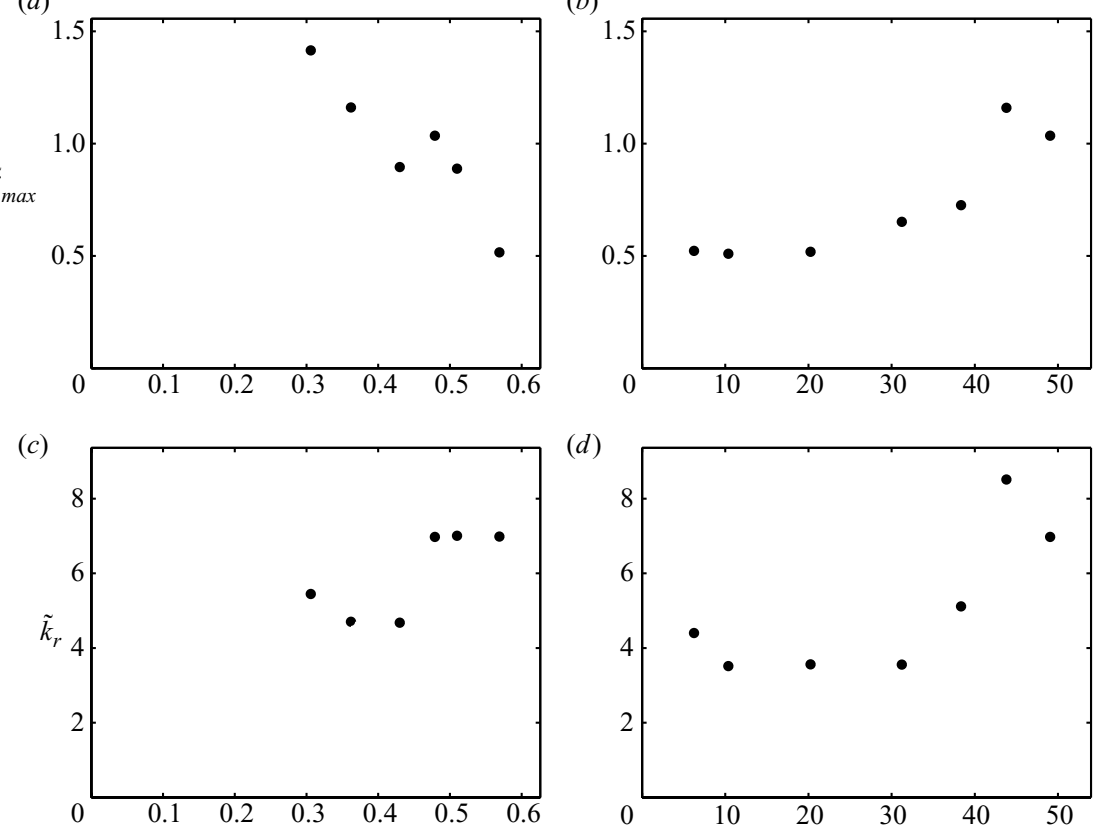

(d)
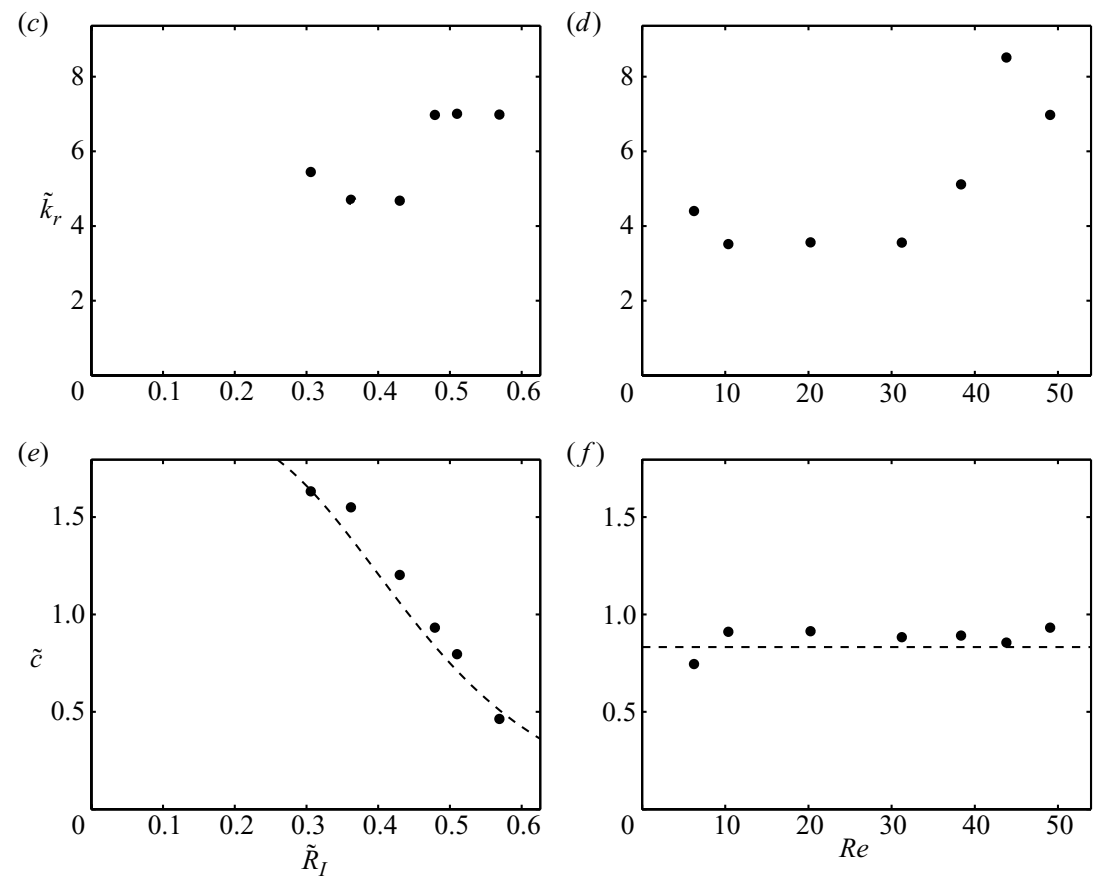

FIGURE 5. $(a, b)$ Frequency of the most amplified mode $\tilde{f}_{\max } .(c, d)$ Real part of the wavenumber at the frequency $\tilde{f}_{\max }, \tilde{k}_{r}\left(\tilde{f}_{\max }\right)$. $(e, f)$ Phase velocity $\tilde{c}\left(\tilde{f}_{\max }\right)$. All the quantities are plotted versus $\tilde{R}_{I}$ at constant $R e=48$ (left column) and versus $R e$ at constant $\tilde{R}_{I}=0.48$ (right column). The dashed lines in $(e)$ and $(f)$ correspond to the theoretical velocity obtained in the short wave regime, $\tilde{c}_{S W}$.

$(e, f)$ : The spatial filtering rate $\alpha$. As expected, $\alpha$ behaves as $-1 / \tilde{L}_{P}$ and accordingly exhibits the same trend as $\tilde{L}_{P}$, with, however, steeper variations at $R_{I} \sim 0.5$ and $R e \sim 35$. We recall that $\alpha$, which gives a local estimation of $\tilde{L}_{P}$, has been introduced to bypass the difficulty in defining the location of the 'entry' in an experimental system. Thereby, $-1 / \alpha$ can a priori reach values lower than the normalized viscous entry length $\tilde{L}_{v}$, but we note that such values $(\alpha<-1)$ have not been obtained in our experiments. Besides, we should point out that, when the parallel base state does not exist anywhere in the system, $\alpha$ may lose its physical meaning. This could also explain a dispersion in the corresponding $\alpha$ measurements (as can be noticed from the values at high $R e$ displayed in figure $6 f$ ). Nevertheless, a steep decrease towards 


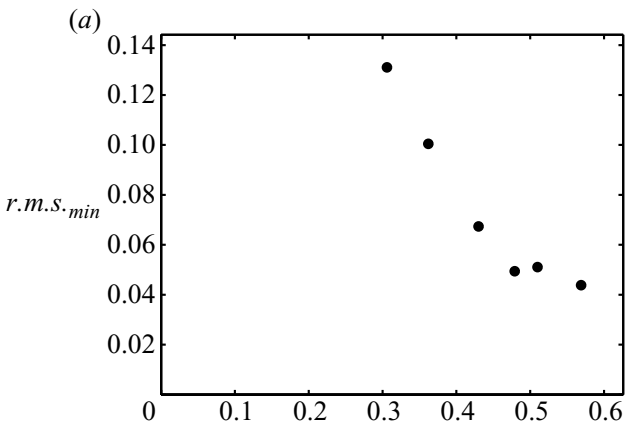

(b)
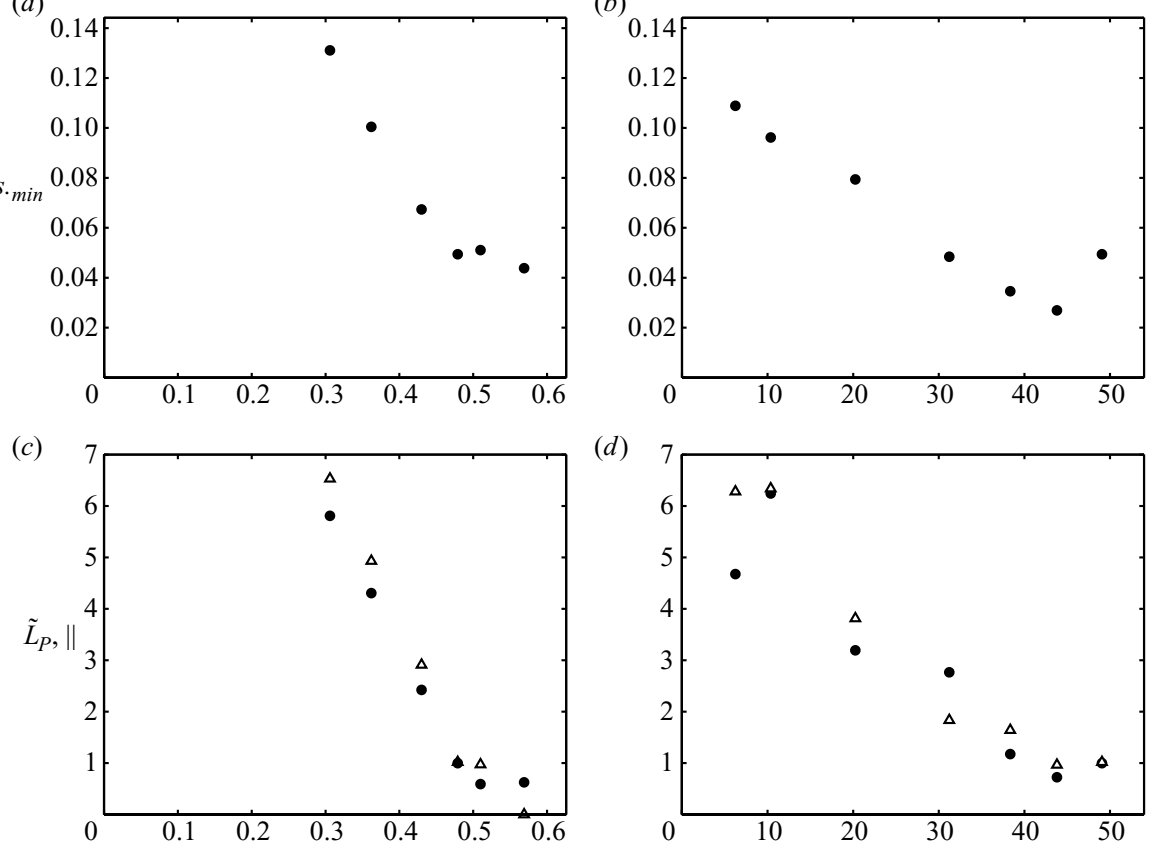

(d)

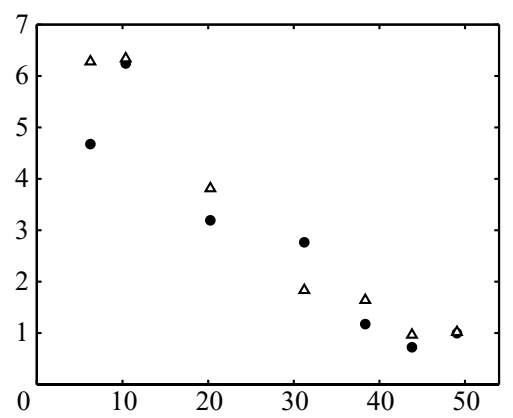

(e)
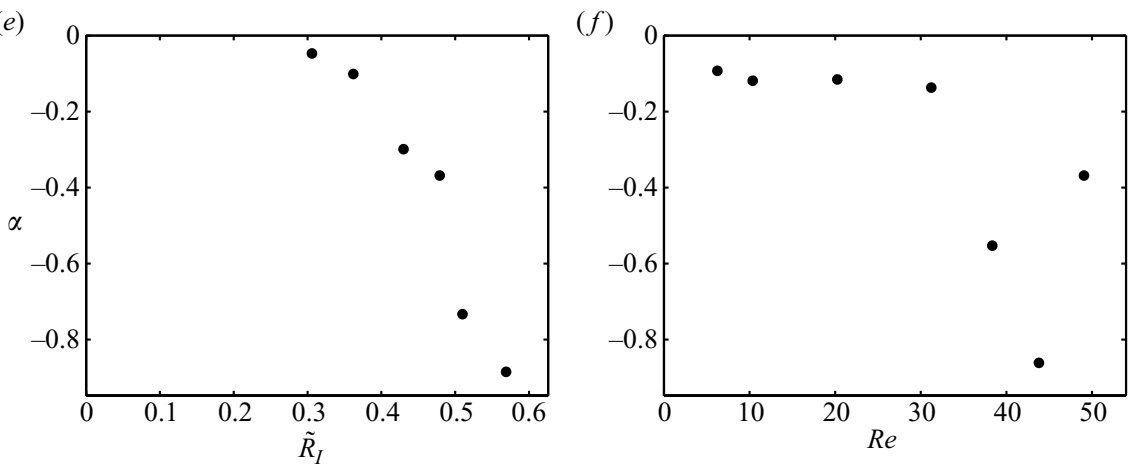

Figure 6. $(a, b)$ Minimum of the r.m.s., r.m.s.min. $(c, d)$ Quasi-parallel length $\tilde{L}_{\|}(\triangle)$ and filtering length $\tilde{L}_{p}(\bullet)$. $(e, f)$ Filtering rate $\alpha$. All the quantities are plotted versus $\tilde{R}_{I}$ at constant $R e=48$ (left column) and versus $R e$ at constant $\tilde{R}_{I}=0.48$ (right column).

low values of $\alpha$ is still a good candidate for the signature of a convective to absolute transition.

To summarize, in this series of figure 6, we clearly observe a strong decrease of the minimum of the frequency r.m.s., of the stable and filtering lengths and of the filtering rate with either $\tilde{R}_{I}$ or $R e$, while keeping the other parameter constant. Although the decreases are steep, the experimental constraints, namely the frequency window width for r.m.s.min and the viscous entry length $\tilde{L}_{v}$ for $\tilde{L}_{\|}, \widetilde{L}_{P}$ and $\alpha$, raise difficulties in measuring the threshold of the transition. Still, the variations of the four measured quantities, presented in figure 6, all support a transition towards an AU state described above $R_{I} \sim 0.45$ and $R e \sim 30$. Although, an agreement for the $R_{I} \sim 0.45$ transition is obtained with both linear stability analysis and nonlinear simulations (Selvam et al. 
(a)

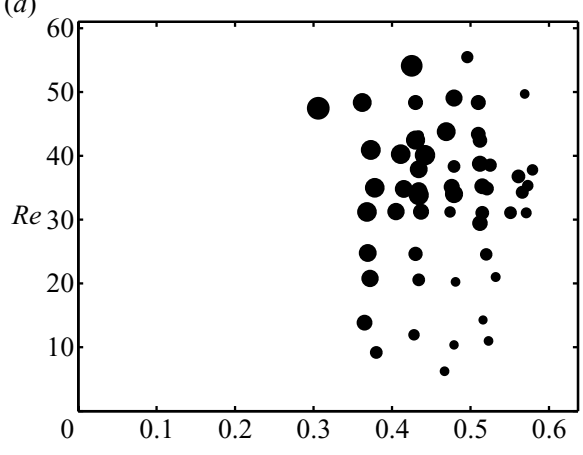

(c)

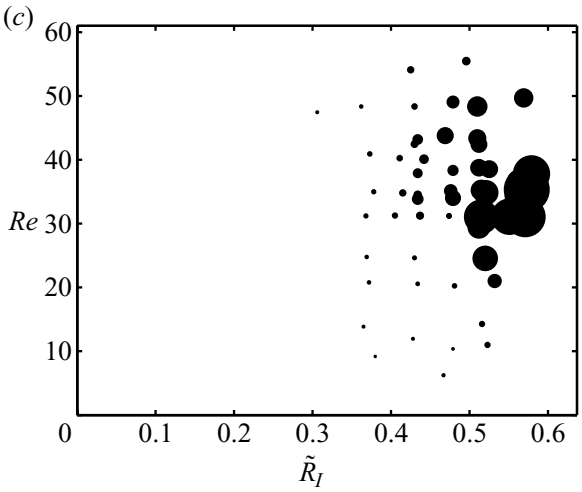

(b)

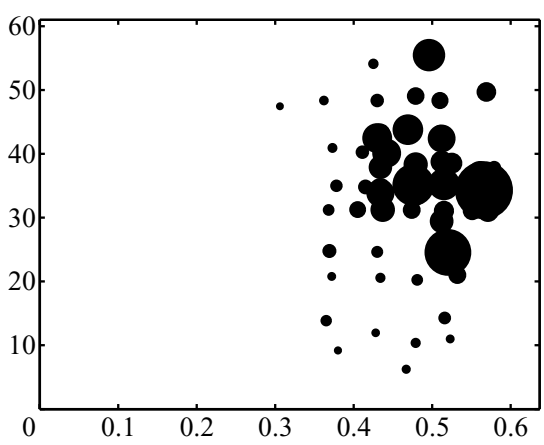

(d)

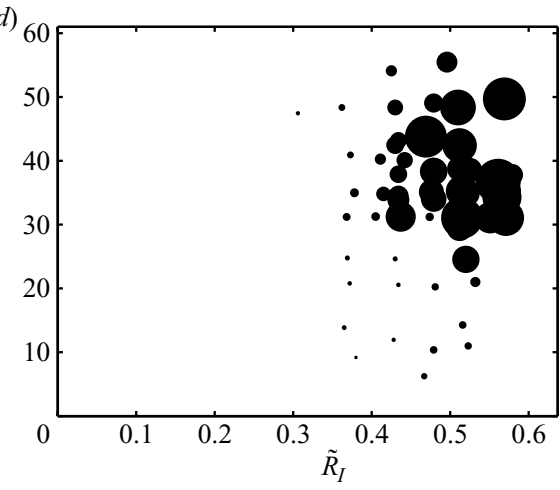

Figure 7. Representation in the $\left(R e-\tilde{R}_{I}\right)$ plane. (a) Frequency of the most amplified mode $\tilde{f}_{\text {max }}$. (b) Inverse of the minimum of the r.m.s., 1/r.m.s.min. (c) Inverse of the filtering length $1 / \tilde{L}_{P} .(d)$ Absolute value of the filtering rate $|\alpha|$. The size of the data points is proportional to the depicted quantity.

2008), our so-obtained transition $R e \sim 30$ is significantly higher than the one obtained numerically $(\operatorname{Re} \sim 10)$.

Figure 7 collects, in the $\left(R e-\tilde{R}_{I}\right)$ plane, all the measurements obtained for: The frequency of the most amplified mode $\tilde{f}_{\max }$ (figure $7 a$ ), the inverse of the minimum of the r.m.s. $1 /$ r.m.s.min, i.e. the quality factor (figure $7 b$ ), the inverse of the filtering length $1 / \tilde{L}_{P}$ (figure $7 c$ ) and the absolute value of the spatial filtering rate $|\alpha|$ (figure $7 d$ ). In each diagram, the size of the data point is proportional to the value of the depicted quantity. As steep increases of $1 /$ r.m.s.min, $\tilde{L}_{P}$ and $|\alpha|$ are expected at the absolute instability transition, absolute states should be pointed out by large dots. Note that the results presented in figures 5 and 6 correspond (in figure 7) to data points on two straight lines parallel to either axis. The general trends obtained from figures 5 and 6 are confirmed in the $\left(R e-\tilde{R}_{I}\right)$ plane of figure 7 . The frequency of the most amplified mode $\tilde{f}_{\max }$ (figure $7 a$ ) increases with $R e$ and decreases with $\tilde{R}_{I}$, but does not exhibit any steep variation. This is not true for the other three quantities displayed in figure 7: The three of them exhibit a steep increase at large $\tilde{R}_{I}$ and $R e$. The corresponding three plots help estimating the reliability of an experimental delineation of the CU/AU states: The frontiers will slightly vary from one plot to the other and with the arbitrary threshold. However, they are very similar and agree qualitatively with the linear stability analysis carried out by Selvam et al. (2008). 
A last check of the $\mathrm{CU} / \mathrm{AU}$ transition accessible with our experimental device is the investigation of the response to a harmonic time perturbation forcing.

\subsection{Response to a time harmonic excitation}

In terms of an oscillator, the response of the system to a disturbance at a given frequency is a key issue (Chomaz, Huerre \& Redekopp 1988). Due to the control of the two pumps by an electronic device (figure 1), the experimental setup enables us to keep the total flow rate constant, while modulating sinusoidally the flow rates of the core and annular fluids. The overall effect of such a forcing is an almost sinusoidal modulation of the interface at the inlet. Figure 8 displays the spatiotemporal and spatio-frequency diagrams obtained at $R e=31$ and $\tilde{R}_{I}=0.48$, for two different forcing frequencies, and also without forcing. In the latter case, the spatio-temporal diagram (figure $8 a$ ) and the spatio-frequency diagram (figure $8 b$ ) are similar to those displayed in figures $4(b)$ and $4(f)$ : A region of parallel flow $\left(\tilde{L}_{\|} \sim 3\right)$ is followed by a region of instability waves, of rather well-defined wavelength and velocity (figure $8 a$ ). Note, however, that the frequency power spectrum of the natural instability has a finite width around the frequency $\tilde{f}_{\text {max }}=0.63$ (figure $8 b$ ). In the presence of forcing at $\tilde{f}_{f}=0.91$ (figures $8 c$ and $8 d$ ), the forced mode, at $\tilde{f}_{f}$, is very narrow, it appears close to the injector, whereas the natural instability still develops beyond a distance of the order of $\tilde{L}_{\|}$, but its amplitude is weakened. This behaviour has been observed experimentally with the forcing frequencies lying outside the natural power spectrum (figure $8 b$ ). On the contrary, when the forcing frequency is close to $\tilde{f}_{\text {max }}$, as for $\tilde{f}_{f}=0.53$ (figures $8 e$ and $8 f$ ), the natural instability disappears. The peak at $\tilde{f}_{f}=0.53$ is narrow (one line in figure $8 f$ which accounts for the regular straight lines in figure 8e), and appears even closer to the injector than for the case $\tilde{f}_{f}=0.91$. One may infer from these experimental observations that, once the forced mode is established, the system reaches a 'stable' bifurcated state. Unlike numerical studies (Selvam et al. 2008), we did observe this behaviour whatever be the values of the control parameters. As a matter of fact, the frequency range in which the forcing annihilates the natural instability is similar to r.m.s.min. Moreover, this range depends on the forcing amplitude (compared to the background noise). We note that this behaviour is compatible with the theoretical predictions by Pier (2003) and the recent experimental results of Hallberg \& Strykowski (2008). These authors showed that the oscillation frequency of an absolute global mode could be selected with a forcing amplitude high enough. Accordingly, conclusive measurements would have required, in our case, a forcing amplitude below the experimental limit. Consequently, the measurements presented here do not provide any improvement for the CU/AU threshold determination.

However, forcing experiments can allow the determination of spatial branches $-\tilde{k}_{i}(\tilde{f})$. In the presence of forcing at $\tilde{f}_{f}$, the logarithm of the forced mode amplitude exhibits a linear part (lying between $\tilde{L}_{v}$ and $\tilde{L}_{\|}$), the slope of which provides $-\tilde{k}_{i}$. Figure 9 shows the so-obtained spatial growth rate $-\tilde{k}_{i}\left(\tilde{f}_{f}\right)$ at $R e=48$ for three values of $\tilde{R}_{I}$. The response of the system to the forcing frequency looks like a resonance curve, which becomes sharper and sharper as $\tilde{R}_{I}$ increases and presents a cusp-like shape for $\tilde{R}_{I}=0.43$. A similar evolution is observed on the curves of figure $9(b)$, as $R e$ is increased at a fixed $\tilde{R}_{I}=0.48$. Note that the results obtained from the natural instability analysis are recovered: As $\tilde{R}_{I}$ is increased at constant $R e$, one observes a decrease of the frequency of the most unstable mode (figures $9 a$ and $5 a$ ) and of the width of the frequency peak (figures $9 a$ and $6 a$ ). As $R e$ is increased at constant $\tilde{R}_{I}$, 
(a)

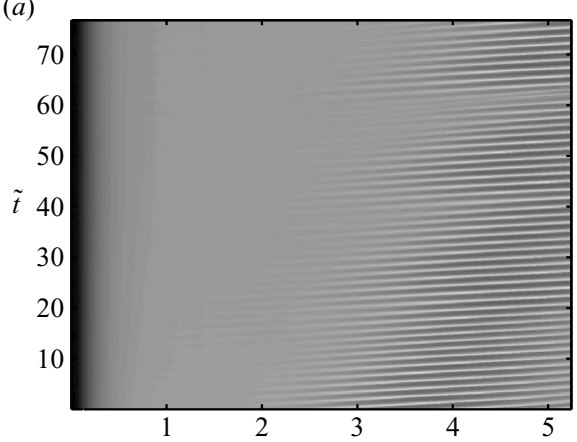

(c)

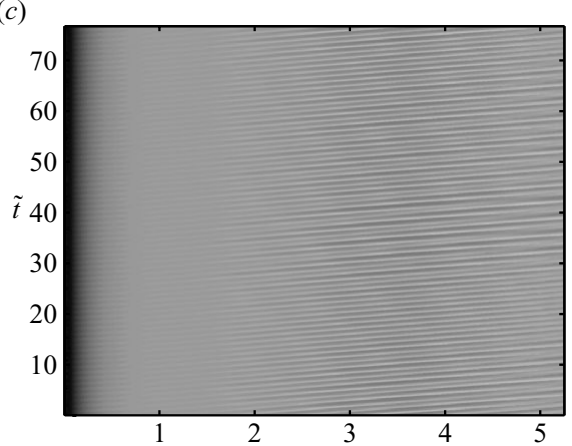

(e)

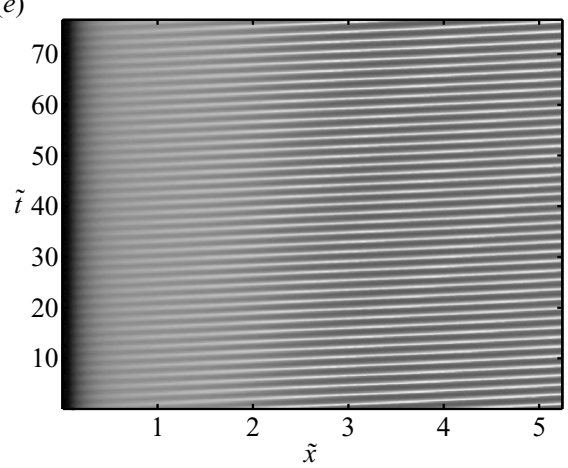

(b)

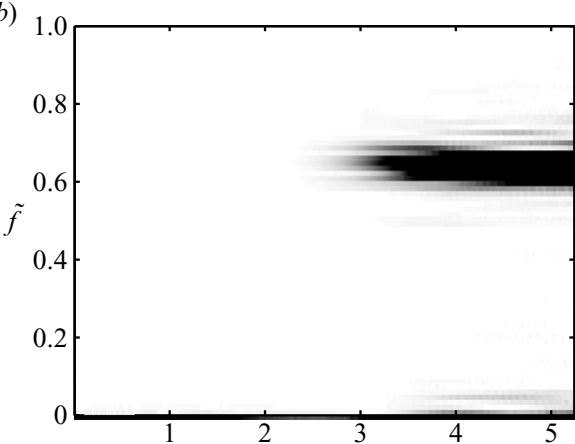

(d)

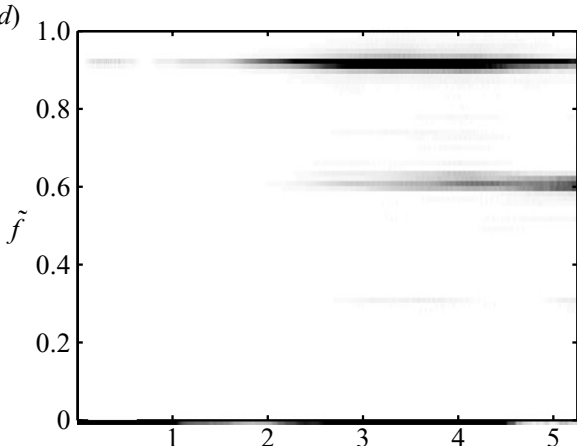

$(f)$

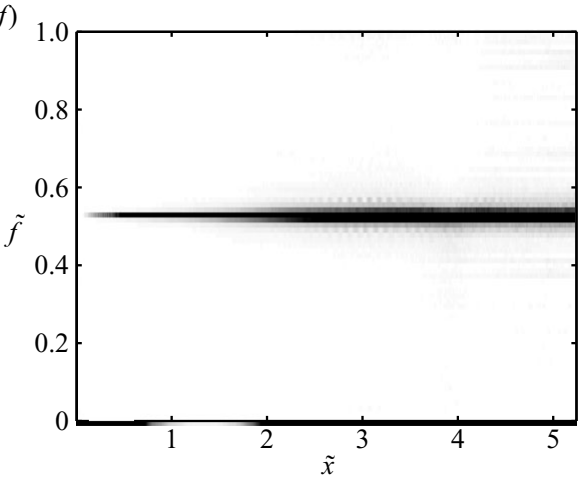

FIGURE 8. Spatio-temporal (left column) and spatio-frequency (right column) diagrams obtained at $R e=31, \tilde{R}_{I}=0.48$, in the absence of forcing $(a, b)$ and with modulation frequencies $\tilde{f}_{f}=0.91(c, d)$ and $\tilde{f}_{f}=0.53(e, f)$. The forced mode appears closer to the entry than the natural mode, $\tilde{f}_{\max }=0.63$. It is either superimposed to the spectrum obtained without modulation $\left(\tilde{f}_{f}=0.91\right)(d)$, or it screens the natural instability $\left(\tilde{f}_{f}=0.53\right)(f)$.

the frequency of the most unstable mode increases (figures $9 b$ and $5 b$ ) and the width of the frequency peak decreases (figures $9 b$ and $6 b$ ).

We could not determine the dispersion curves for values larger than $R e=31$ and $\tilde{R}_{I}=0.43$, as $\tilde{L}_{\|}$, and thus the linear region, becomes too small to enable reliable measurements. The vicinity of the threshold is once again difficult to investigate. Nevertheless, according to Huerre \& Rossi (1998), the appearance of a cusp should be taken as a strong warning signal of a pinch of the two spatial branches $\tilde{k}_{i}^{+}(\tilde{f})$ and $\tilde{k}_{i}^{-}(\tilde{f})$. This infers that a non-cuspy dispersion curve is a proof for a CU state. This is 


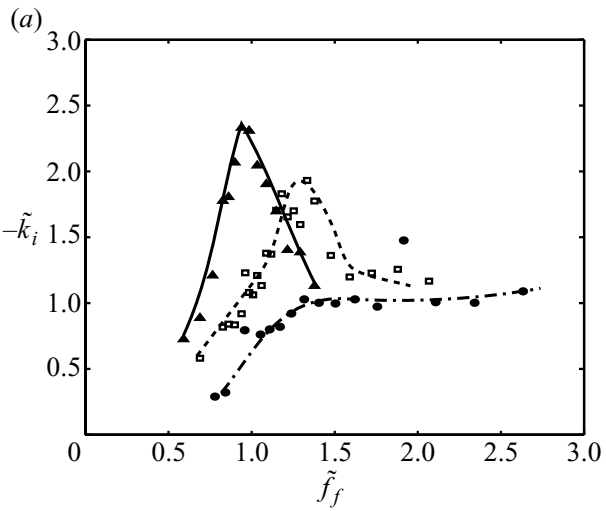

(b)

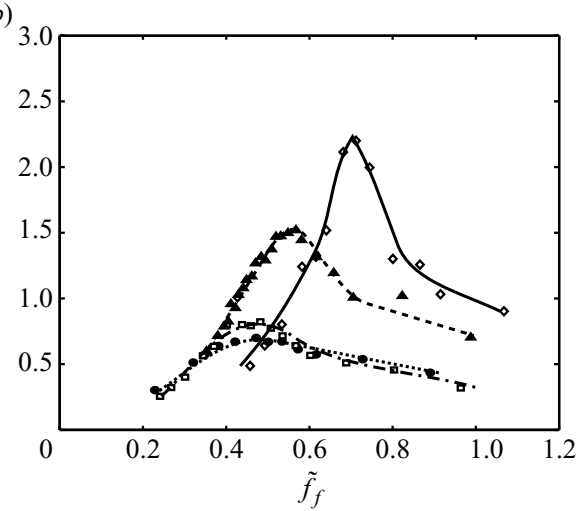

FiguRE 9. Spatial growth rate $-\tilde{k}_{i}$ versus the forcing frequency $\tilde{f}_{f}$. (a) Constant $R e=48$ for different $\tilde{R}_{I}\left(\tilde{R}_{I}=0.30(\bullet), \tilde{R}_{I}=0.36(\square), \tilde{R}_{I}=0.43(\mathbf{\Delta})\right)$. (b) Constant $\tilde{R}_{I}=0.48$ for different $R e$ values $(R e=6(\bullet), R e=10(\square), R e=20(\Delta), R e=31(\diamond))$. The lines are guides to the eye.

the case for the dispersion curves measured for $\tilde{R}_{I} \leqslant 0.36$ at $\operatorname{Re}=48$ and for $\operatorname{Re} \leqslant 20$ at $\tilde{R}_{I}=0.48$, which gives, respectively, two lower bounds of the transition. Moreover, one can suspect from figure 9 that the thresholds are close to $\tilde{R}_{I} \sim 0.43$ at $R e=48$ and $R e \sim 31$ at $\tilde{R}_{I}=0.48$. These transitions are comparable with the estimations obtained from figure 6 .

\section{Discussion/Conclusions}

We performed experiments of miscible non-buoyant core-annular flows, which always developed an axisymmetric instability. Some features of the resulting flows suggested that they might be either convectively unstable or absolutely unstable, depending on the control parameters $R e$ and $\tilde{R}_{I}$. We have attempted to delineate experimentally the transition $\mathrm{CU} / \mathrm{AU}$. The results obtained from the present experimental work may be summarized as follows.

The standard measurements used to characterize instabilities, such as the wavelength, the frequency and the wave speed of small perturbations of the pseudointerface, do not exhibit any steep variation which could indicate the transition. And yet, a jump could have been expected, as the selection process differs from CU to AU states. Indeed, the most spatially unstable mode, which dominates in a CU state should differ from the absolute mode, observed in an AU state. Actually, although different, these two modes merge at the transition. Therefore, the latter should not correspond to a discontinuity, but to a change of slope which is difficult to localize on the experimental curves characterizing the evolution of the most amplified wave (figure 5).

As an AU system behaves like a self-frequency resonator, we have analysed the frequency content of our system in terms of the minimum of the frequency width r.m.s.min, the filtering length $\widetilde{L}_{P}$ (which was found almost identical to the quasi-parallel length $\tilde{L}_{\|}$) and the rate of filtering $\alpha$. As a result, r.m.s.min $, \tilde{L}_{P}, \tilde{L}_{\|}$and $\alpha$ are good indicators of the states which are likely to be AU, at large values of $\tilde{R}_{I}$ and $R e$. But because their evolutions are relatively smooth, we have difficulties in determining experimentally a threshold for the transition. 
(a)

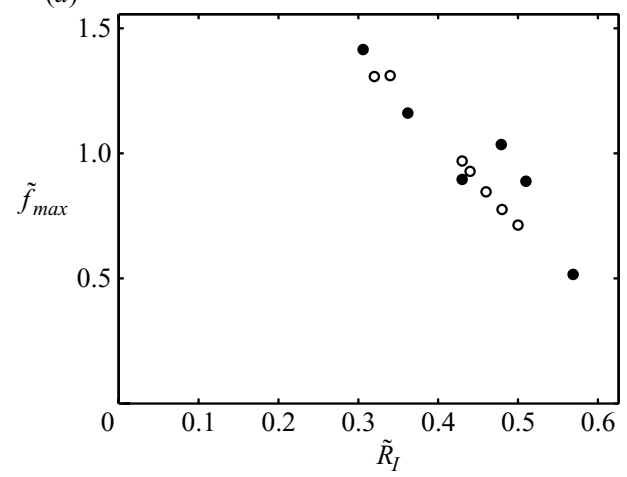

(b)

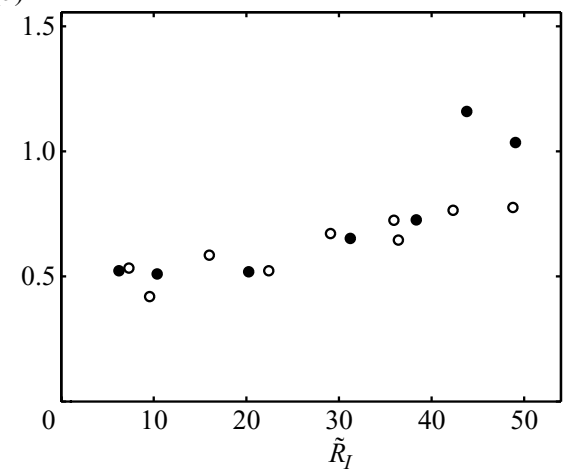

FIGURE 10 . Effect of the injection nozzle radius $\tilde{R}_{n}$ on the frequency $\tilde{f}_{\max }\left(\tilde{R}_{n}=0.75(\bullet)\right.$ and $\tilde{R}_{n}=0.5(\bigcirc)$ ). (a) At constant $R e=48$, varying $\tilde{R}_{I}$. (b) At constant $\widetilde{R}_{I}=0.48$, varying $R e$.

According to the theoretical approach (see Couairon \& Chomaz 1997a,b), the healing length, which denotes the length needed for a global mode to grow from the inlet, is infinite at the absolute transition, and decreases with the distance to criticality. This property should provide a good criterion to analyse the evolution of either $\tilde{L}_{P}$ or $\tilde{L}_{\|}$. However, it is important to stress the reasons why the lengths measured in experiments may not be comparable with the healing length, defined in a theoretical context. Firstly, the latter was introduced considering a semi-infinite domain, for which the base state holds at $\tilde{x}=0$. In our experiments, the boundary conditions are perturbed by the viscous entry length. Secondly, the characterization of the healing length assumes that no noise is injected in the system, once the latter has been initially perturbed. In the presence of a permanent noise, any CU system (AU or not) will amplify it over a typical finite length. As a matter of fact, $\tilde{L}_{P}$ and $\tilde{L}_{\|}$do not diverge as the control parameters approach the transition CU/AU.

Nevertheless, the analysis of the response of the system to a time harmonic forcing has enabled us to obtain the spatial branches of the dispersion curve. And the cusplike shape of these curves, which become sharper and sharper in the area of large radius and high Reynolds numbers is a strong warning of the occurrence of an absolute region.

Theoretical analyses for non-parallel real flows have shown that in some absolute unstable states (for the so-called pulled fronts), global modes may be selected by the linear dispersion relation at the inlet $(\tilde{x}=0)$ (see Couairon \& Chomaz 1997a, $b$; Chomaz 2003). In such a case, the inlet conditions should control the instability features (frequency, healing length, etc.). On the contrary, in a CU state, these features are controlled by the noise. Therefore, one can expect a different response of the two states to a change in the boundary conditions. This can be experimentally achieved by using different inlet nozzles. Figure 10 shows the evolution of the frequency $\tilde{f}_{\max }$ of the most amplified mode, for a constant $R e=48$ and different $\tilde{R}_{I}$ (figure $10 a$ ) and for a constant $\tilde{R}_{I}=0.48$ and different $R e$ (figure $10 \mathrm{~b}$ ), for two nozzles with different inner radius, $\tilde{R}_{n}=0.5$ and $\tilde{R}_{n}=0.75$. We observe that the selected frequency is almost the same for small $R e$ and $\tilde{R}_{I}$ but rather different for higher values. From this observation one could have inferred that the system is AU when the frequency is inlet dependent. Unfortunately, the frequency mismatch is obtained in experiments for which $\widetilde{L}_{\|} \sim \tilde{L}_{v}$ (see figure 6). Hence, the amplification of the noise is totally achieved in the viscous entry length $\tilde{L}_{v}$, where the dispersion curve depends on the injection 
nozzle. Consequently, a frequency mismatch can be observed even in a CU state and no conclusion can be drawn from figure 10.

In conclusion, r.m.s.min $, \tilde{L}_{P}, \tilde{L}_{\|}$and $\alpha$ behaviours and the evolution of the cusplike shape of the forced spatial growth rate build a body of evidence for an absolute unstable region to develop at high $\tilde{R}_{I}$ and high $R e$ numbers. A more quantitative determination of the transition threshold is not achievable due to the inherent imperfection of experiments. These experimental results are discussed and compared to linear stability results and nonlinear numerical simulations in the following companion paper of Selvam et al. (2008). In this paper, it is shown that the $\mathrm{CU} / \mathrm{AU}$ transition is indeed very close to our experimental results.

The authors would like to thank E. Meiburg and B. Selvam for fruitful collaboration. M. d'Olce is supported by a $\mathrm{PhD}$ grant from the french Ministère de l'Enseignement Supérieur et de la Recherche. L. Talon was partially funded by the French Ministry of Foreign Affairs through the Lavoisier fellowship program.

\section{REFERENCES}

Albert, F. \& Charru, F. 2000 Small Reynolds number instabilities in two-layer Couette flow. Eur. J. Mech. B-Fluids 19, 229-256.

BaI, R., Chen, K. \& Joseph, D. D. 1992 Lubricated pipelining: stability of core-annular flow. Part 5. Experiments and comparison with theory. J. Fluid Mech. 240, 97-132.

Balasubramaniam, R., Rashidnia, N., Maxworthy, T. \& Kuang, J. 2005 Instability of miscible interfaces in a cylindrical tube. Phys. Fluids 17, 052103.

Brevdo, L., LAURE, P. \& Bridges, T. J. 1999 Linear pulse structure and signalling in a film flow on an inclined plane. J. Fluid Mech. 396, 37-71.

BriggS, R. J. 1964 Electron-Stream Interaction with Plasma. MIT Press.

CaO, Q., Ventresca, A. L., Sreenivas, K. R. \& Prasad, A. K. 2003 Instability due to viscosity stratification downstream of a centerline injector. Can. J. Chem. 81, 913-922.

Charru, F. \& Hinch, E. J. 2000 'Phase diagram' of interfacial instabilities in a two-layer Couette flow and mechanism for the long-wave instability. J. Fluid Mech. 414, 195-223.

Chen, C.-Y. \& Meiburg, E. 1996 Miscible displacement in capillary tubes. Part 2. Numerical simulations. J. Fluid Mech. 326, 57-67.

Chomaz, J.-M. 2003 Fully nonlinear dynamics of parallel wakes. J. Fluid Mech. 495, 57-75.

Chomaz, J.-M. 2005 Global instabilities in spatially developing flows: non-normality and nonlinearity. Annu. Rev. Fluid Mech. 37, 357-392.

Chomaz, J.-M., Huerre, P. \& Redekopp, L. G. 1988 Bifurcations to local and global modes in spatially developing flows. Phys. Rev. Lett. 60, 25-28.

Couarron, A. \& Chomaz, J.-M. 1997a Absolute and convective instabilities, front velocities and global modes in nonlinear systems. Phys. D 108, 236-276.

Couairon, A. \& Chomaz, J.-M. $1997 b$ Pattern selection in the presence of a cross flow. Phys. Rev. Lett. 79, 2666-2669.

Duprat, C., Ruyer-Quil, C., Kalliadasis, S. \& Giorgiutti-Dauphine, F. 2007 Absolute and convective instabilities of a viscous film flowing down a vertical fiber. Phys. Rev. Lett. 98, 244502.

Gondret, P., Ern, P., Meignin, L. \& Rabaud, M. 1999 Experimental evidence of a nonlinear transition from convective to absolute instability. Phys. Rev. Lett. 82, 1442-1445.

Guillot, P., Colin, A., Utada, A. S. \& Adjari, A. 2007 Stability of a jet in confined pressure-driven biphasic flows at low Reynolds numbers. Phys. Rev. Lett. 99, 104502.

Hallberg, M. P. \& Strykowski, P. J. 2008 Open-loop control of fully nonlinear self-excited oscillations. Phys. Fluids 20, 041703.

Hickox, C. E. 1971 Instability due to viscosity and density stratification in axisymmetric pipe flow. Phys. Fluids 14, 251. 
Hinch, E. J. 1984 A note on the mechanism of the instability at the interface between two shearing fluids. J. Fluid Mech. 144, 463-465.

Hu, H. H. \& JosePh, D. D. 1989 Lubricated pipelining: stability of core-annular flow. Part 2. J. Fluid Mech. 205, 395-396.

Huerre, P. \& Monkewitz, P. A. 1990 Local and global instabilities in spatially developing flows. Annu. Rev. Fluid Mech. 22, 473-537.

Huerre, P. \& Rossi, M. 1998 Hydrodynamic instabilities in open flows. In Hydrodynamics and Nonlinear Instabilities (ed. C. Godreche \& P. Manneville). Cambridge University Press.

Joseph, D. D., Bai, R., Chen, K. P. \& Renardy, Y. Y. 1997 Core-annular flows. Ann. Rev. Fluid Mech. 29, 65-90.

Joseph, D. D. \& Renardy, Y. Y. 1992 a Fundamentals of Two-Fluid Dynamics. Part I. Mathematical Theory and Applications. Springer-Verlag.

Joseph, D. D. \& Renardy, Y. Y. 1992 b Fundamentals of Two-Fluid Dynamics. Part II. Lubrificated Transport, Drops and Miscible Liquids. Springer-Verlag.

Joseph, D. D., Renardy, M. \& Renardy, Y. 1984 Instability of the flow of two immiscible liquids with different viscosities in a pipe. J. Fluid Mech. 141, 309-317.

Kouris, C. \& Tsamopoulos, J. 2001 Dynamics of axisymmetric core-annular flow in a straight tube. I. The more viscous fluid in the core, bamboo waves. Phys. Fluids 13, 841-858.

Kouris, C. \& Tsamopoulos, J. 2002 Dynamics of the axisymmetric core-annular flow. II. The less viscous fluid in the core, saw tooth waves. Phys. Fluids 14, 1011-1029.

Kuang, J., Maxworthy, T. \& Petitjeans, P. 2003 Miscible displacements between silicone oils in capillary tubes. Eur. J. Mech. B 22, 271-277.

Le Bars, M. \& Davaille, A. 2002 Stability of thermal convection in two superimposed miscible viscous fluids. J. Fluid Mech. 471, 339-363.

D’Olce, M., Martin, J., Rakotomalala, N., Salin, D. \& Talon, L. 2008 Pearl and mushroom instability patterns in two miscible fluids core annular flow. Phys. Fluids 20, 24104.

Papageorgiou, D. T., Maldarelli, C. \& Rumschitzki, D. S. 1990 Nonlinear interfacial stability of core-annular film flows. Phys. Fluids 2, 340-352.

Petitjeans, P. \& Maxworthy, T. 1996 Miscible displacements in capillary tubes. Part 1. Experiments. J. Fluid Mech. 326, 37-56.

PIER, B. 2003 Open-loop control of absolutely unstable domains. Proc. R. Soc. London Se A 459, $1105-1115$.

Scoffoni, J., Lajeunesse, E. \& Homsy, G. M. 2001 Interface instabilities during displacement of two miscible fluids in a vertical pipe. Phys. Fluids 13, 553-556.

Selvam, B., Merk, S., Govindarajan, R. \& Meiburg, E. 2007 Stability of miscible core-annular flow with viscosity stratification. J. Fluid Mech. 592, 23-49.

Selvam, B., Talon, L., Lesshafft, L. \& Meiburg, E. 2009 Convective/absolute instability in miscible core-annular flow. Part 2. Numerical simulation and nonlinear global modes. J. Fluid Mech. 618, 323-348.

ToRrest, R. S. 1982 Rheological properties of aqueous solutions of the polymer natrosol $250 \mathrm{hhr}$. J. Rheol. 26, 143-151. 